\begin{tabular}{|l|l|l|l|l|}
\hline$\wedge$ & PMIC & pmic201500202 & Dispatch: December 9, 2015 & CE: \\
\cline { 2 - 5 } & Journal & MSP No. & No. of pages: 11 & PE: XXXXX \\
\hline
\end{tabular}

\title{
ITRAQ proteomics analysis reveals that PI3k is highly associated with bupivacaine-induced neurotoxicity pathways
}

\author{
Wei Zhao ${ }^{1 * *}$, Zhongjie Liu ${ }^{1 * *}$, Xujiao Yu ${ }^{1}$, Luying Lai ${ }^{1}$, Haobo Li ${ }^{2}$, Zipeng Liu ${ }^{2}$ Le Li ${ }^{1}$, \\ Shan Jiang ${ }^{1}$, Zhengyuan $\mathrm{Xia}^{2 *}$ and Shi-yuan $\mathrm{Xu}^{1}$ \\ ${ }^{1}$ Department of Anesthesiology, Zhujiang Hospital, Southern Medical University, Guangdong Province, China \\ ${ }^{2}$ Li Ka Shing Faculty of Medicine, University of Hong Kong, Pokfulam, Hong Kong \\ 2 Department of Anesthesiology, the Second Affiliated Hospital \& Yuying Children's Hospital of Wenzhou Medical University, \\ Wenzhou, Zhejiang, China
}

Bupivacaine, a commonly used local anesthetic, has potential neurotoxicity through diverse signaling pathways. However, the key mechanism of bupivacaine-induced neurotoxicity remains unclear. Cultured human SH-SY5Y neuroblastoma cells were treated (bupivacaine) or untreated (control) with bupivacaine for $24 \mathrm{~h}$. Compared to the control group, bupivacaine significantly increased cyto-inhibition, cellular reactive oxygen species, DNA damage, mitochondrial injury, apoptosis (increased TUNEL-positive cells, cleaved caspase 3, and Bcl-2/Bax), and activated autophagy (enhanced LC3II/LC3I ratio). To explore changes in protein expression and intercommunication among the pathways involved in bupivacaine-induced neurotoxicity, an 8-plex iTRAQ proteomic technique and bioinformatics analysis were performed. Compared to the control group, 241 differentially expressed proteins were identified, of which, 145 were up-regulated and 96 were down-regulated. Bioinformatics analysis of the cross-talk between the significant proteins with altered expression in bupivacaine-induced neurotoxicity indicated that phosphatidyl-3-kinase (PI3K) was the most frequently targeted protein in each of the interactions. We further confirmed these results by determining the downstream targets of the identified signaling pathways (PI3K, Akt, FoxO1, Erk, and JNK). In conclusion, our study demonstrated that PI3K may play a central role in contacting and regulating the signaling pathways that contribute to bupivacaine-induced neurotoxicity.

Keywords:

Bupivacaine / Itraq / Local anesthetic / Neurotoxicity / PI3K

Additional supporting information may be found in the online version of this article at the publisher's web-site

\section{Introduction}

Local anesthetics (LAs) are necessary for epidural anesthesia, spinal anesthesia, peripheral nerve blocks, and pain manage-

Correspondence: Shiyuan $\mathrm{Xu}$, Department of Anesthesiology, Zhujiang Hospital, Southern Medical University, 253 Industrial Road, Guangzhou City, Guangdong Province, China

E-mail: xushiyuan355@163.com

Abbreviations: DHE, dihydroethidium; iTRAQ, Isobaric tagging for relative and absolute protein quantification; LA, local anesthetics; LDH, Lactate Dehydrogenase; MAPK, mitogen-activated protein kinase; mTOR, Mammalian target of rapamyc; NAC, Nacetyl cysteine; PI3K, Phosphoinositide3-kinases; ROS, reactive oxygen species

(C) 2015 WILEY-VCH Verlag GmbH \& Co. KGaA, Weinheim
Received: May 27, 2015

Revised: October 26, 2015

Accepted: November 14, 2015 ment, but the continuous application of a high concentration of LA or a long-term exposure to LA can cause neurotoxicity [1-3]. Although the incidence of neurotoxicity due to the application of clinical concentrations of LAs is rare, it is a severe problem once it occurs. Epidemiology surveys suggest that complications, such as transient neurologic syndrome (TNS), may occur in more than $30 \%$ of patients who receive spinal anesthetics. Serious complications, such as cauda equina syndrome, affect approximately 1 in 8000 patients [4-7].

Bupivacaine, an amide-type local anesthetic, is one of the most widely used local anesthetics in clinics. Studies in

\footnotetext{
*Additional corresponding author: Dr. Zhengyuan Xia E-mail: zyxia@hku.hk

** Wei Zhao and Zhongiie Liu have contributed equally to this work. Colour Online: See the article online to view Figs. 1-4 in colour.
} 
animal and cellular models have shown that bupivacaine can be neurotoxic when applied to neural tissues at clinical concentrations $[1,8,9]$. Multiple factors contribute to the neurotoxicity of bupivacaine. The generation of excess reactive oxygen species (ROS) [10], which are normal by-products of cellular metabolism, can destabilize intracellular calcium homeostasis [11], activate MAPKs [12] and may be involved in the pathogenesis of bupivacaine-induced apoptosis. Lirk et al. demonstrated that bupivacaine-induced apoptosis involves the generation of ROS in Schwann cells and activates p38-MAPK in dorsal root ganglion (DRG) neurons. A recent study showed that ROS over-production, concomitant with the inhibition of the Akt/mTOR/p70S6K signaling pathway, contributed to Streptococcus pneumoniae-induced autophagy (a programmed cell death pathway) in A549 Cells [13]. We recently demonstrated that bupivacaine-induced ROS generation was a mechanism by which cellular toxicity was produced [14]. Furthermore, impaired autophagosome clearance contributes to bupivacaine-induced myotoxicity in mouse myoblasts [9]. All of these studies indicate that bupivacaine may induce neurotoxicity primarily through autophagy and apoptosis. It has been shown that there is a frequent interplay between autophagy and apoptosis [15]. However, to date, there are no systematic studies regarding the potential interplay between autophagy and apoptosis nor are there any studies on the underlying mechanism governing this interplay in bupivacaine-induced neurotoxicity.

In the present study, we examined bupivacaine-induced neurotoxicity in vitro in SH-SY5Y cells, an undifferentiated human neuroblastoma cell line $[16,17]$. We used isobaric tagging for relative and absolute protein quantification (iTRAQ) [18-20], a powerful proteomics method developed to detect the changes in protein expression in different biological samples, to explore the changes in protein expression and intercommunication in bupivacaine-induced neurotoxicity related pathways. The significant changes and connections in signaling pathways identified by bioinformatics analysis were tested/confirmed in cell models of bupivacaine-induced neurotoxicity.

\section{Materials and methods}

\subsection{Reagents}

Bupivacaine was purchased from Sigma, USA. DMEM-F12, fetal calf serum and pancreatic enzyme (including or excluding) EDTA were purchased from Gibco, USA. SH-SY5Y cells were obtained from the cell bank of the Chinese Academy of Sciences (Shanghai, China).

\subsection{Cell culture and treatment}

SH-SY5Y, an undifferentiated human neuroblastoma cell line, was grown in DMEM-F12 (Gibco, USA) supplemented with $10 \%$ fetal bovine serum, penicillin $(100 \mathrm{U} / \mathrm{mL})$ and streptomycin $(100 \mu \mathrm{g} / \mathrm{mL})$ in a humidified 5\% CO2 atmosphere incubator at $37^{\circ} \mathrm{C}$. The culture medium was renewed once a day during cell growth. After seeding onto plates for $24 \mathrm{~h}$, cells were exposed to bupivacaine for $24 \mathrm{~h}$.

\subsection{Measurement of cell viability using the CCK-8 assay}

SH-SY5Y cells were seeded at a density of $1 \times 10^{4}$ cells per well in a 96-well cell culture plate, and cell viability was determined with the Cell Counting Kit-8 (CCK8, Dojindo, Japan) according to the manufacturer's instructions. Briefly, after the cells were exposed to bupivacaine $(0,0.5,1.0,1.5,2.0$, and $2.5 \mathrm{mM})$ for $24 \mathrm{~h}$, CCK-8 $(10 \mu \mathrm{L} /$ well $)$ was added and incubated for an additional $4 \mathrm{~h}$ at $37^{\circ} \mathrm{C}$, according to the instructions provided by manufacturer. The optical density of the homogeneous purple solutions was measured at $450 \mathrm{~nm}$ using a spectrophotometer. In parallel, cells were cultured in DMEM-F12 medium under the same conditions. Each experiment was performed in triplicate, and the viability of the control group without bupivacaine treatment was set as $100 \%$; the other groups were normalized to the corresponding control values.

\subsection{Hoechst 33258 fluorescence detection}

Cells were seeded onto 24 -well plates at $1 \times 10^{5}$ cells/well in $500 \mu \mathrm{L}$ culture medium and assigned to two groups: (i) untreated control (Con) and (ii) cells treated with $1.5 \mathrm{mM}$ bupivacaine for $24 \mathrm{~h}$ (Bup). After incubation, fluorescent microscopy analysis was performed using Hoechst 33258 staining (Sigma, USA) to detect cell apoptosis, a process during which the dye enters the cell and changes in the target cell nucleus and DNA damage occur. The images were observed and captured with a fluorescent microscope (BX41System microscope; Olympus, DP72 digital camera) at the $340 \mathrm{~nm}$ excitation wavelength (UV-light) as previously described [21].

\subsection{Determination of Cellular Injury}

Cells were seeded onto 6 -well plates at $5 \times 10^{5}$ cells/well in $2 \mathrm{~mL}$ culture medium. Briefly, after the cells were exposed to bupivacaine $(0,0.5,1.0,1.5,2.0$, and $2.5 \mathrm{mM})$ for $24 \mathrm{~h}$, the lactate dehydrogenase (LDH) content was measured with an LDH Cytotoxicity Assay Kit (Roche, Indianapolis, USA) as described [22].

Cells were seeded onto 24 -well plates at $1 \times 10^{5}$ cells/well in $500 \mu \mathrm{L}$ culture medium and assigned to two groups: (i) untreated control (Con) and (ii) cells treated with $1.5 \mathrm{mM}$ bupivacaine for $24 \mathrm{~h}$ (Bup). Cell apoptosis was detected by double immunofluorescence staining of TUNEL using the In Situ Cell Death Detection Kit (Roche, Indianapolis, USA) as 
previously described [22]. Mitochondrial membrane potential (MMP) loss was measured using a JC-1 mitochondrial membrane potential assay kit (Cayman chemical) as described [23]. The images were observed and captured with a fluorescence microscope (BX41System microscope; Olympus, DP72 digital camera).

\subsection{Comet assay}

A single-cell gel electrophoresis assay (comet assay) was applied to measure DNA damage including double-strand breaks, single strand breaks, alkali labile sites, and oxidative DNA base damage in individual cells. The SH-SY5Y cells $(2 \times$ $10^{5}$ cells) were plated in 6-well plates, and the extent of DNA damage was measured using a kit (Trevigen's Comet Assay ${ }^{\circledR}$ Kit), based on the manufacturer's instructions. The images were observed and captured with a fluorescence microscope (BX41System microscope; Olympus, DP72 digital camera) at 200x

magnification. A minimum of 50 randomly selected cells (from each of the two replicate slides) were analyzed with the Comet Assay Software Project (CASP-6.0, University of Wroclaw, Poland).

\subsection{Measurement of Cellular ROS in Cultured SH-SY5Y cells}

Cells were seeded onto 6 -well plates at $5 \times 10^{5}$ cells/well in $2 \mathrm{~mL}$ culture medium. Superoxide generation in SH-SY5Y cells was estimated by DHE staining as previously described [22]. Briefly, cells were loaded with DHE at a concentration of $10 \mu \mathrm{M}$ for $30 \mathrm{~min}$ at $37^{\circ} \mathrm{C}$. The images were observed and captured under a fluorescence microscope (BX41System microscope; Olympus, DP72 digital camera). The DHE fluorescence of DHE-labeled positive nuclei were calculated in each of five randomly selected fields and were expressed as a percentage of the DHE-stained positive nuclei compared with the controls by a quantitative morphometric method.

\subsection{Western blotting}

Cells were seeded onto 6 -well plates at $5 \times 10^{5}$ cells/well in $500 \mu \mathrm{L}$ culture medium and assigned to two groups: (i) untreated control (Con) and (ii) cells treated with $1.5 \mathrm{mM}$ bupivacaine for $24 \mathrm{~h}$ (Bup). After treatment, cells were lysed in lysis buffer to extract the proteins. After centrifugation, the protein was collected, and the protein concentration was determined by the Bradford method for Western blotting. Equal amounts of protein extracts were separated by $10 \%$ SDS-PAGE and transferred to PVDF membranes (Immobilon-P, Millipore, Bedford, MA, USA). The blots were blocked with 5\% milk and incubated overnight at $4^{\circ} \mathrm{C}$ with antibodies against caspase-3 rabbit, 1:1000; Cell Signaling cleaved-caspase3 rabbit, 1:1000;
Cell Signaling), Bax (rabbit, 1:1000; Cell Signaling), bcl-2 (rabbit, 1:1000; Cell Signaling), LC3-I/II (rabbit, 1:1000; Cell Signaling), $\beta$-tubulin (rabbit, 1:3000; Cell Signaling), PI3K, Akt, p-Akt, FoxO1, p-FoxO1, JNK, P-JNK ERK and p-ERK (rabbit, 1:1000; Cell Signaling). These blots were further incubated with HRP-conjugated secondary antibody, developed in ECL solution, and exposed onto hyperfilm (Amersham Biosciences) for 1-10 min. The intensity of the selected bands was captured and analyzed using Image J software (NIH).

\subsection{ITRAQ}

\subsubsection{Protein preparation}

The SH-SY5Y cells exposed (as experimental group) or not exposed (as control group) to $1.5 \mathrm{mM}$ bupivacaine were resuspended in lysis buffer (7 M urea, $2 \mathrm{M}$ thiourea, $4 \%$ CHAPS, $40 \mathrm{mM}$ Tris- $\mathrm{HCl}, \mathrm{pH} 8.5,1 \mathrm{mM}$ PMSF, and 2 mM EDTA) and sonicated on ice. Three biological replicates were performed for each group. The control group samples were named C1, $\mathrm{C} 2$, and $\mathrm{C} 3$. The experimental group samples were named B1, B2, and B3. The proteins were reduced with $10 \mathrm{mM}$ DTT (final concentration) at $56^{\circ} \mathrm{C}$ for $1 \mathrm{~h}$ and then alkylated by $55 \mathrm{mM}$ IAM (final concentration) in the darkroom for $1 \mathrm{~h}$. After centrifuging at $30000 \times \mathrm{g}$ at $4^{\circ} \mathrm{C}$, an aliquot of the supernatant was used to determine the protein concentration by the Bradford method.

\subsubsection{ITRAQ labeling and SCX fractionation}

Protein $(100 \mu \mathrm{g})$ was removed from each sample solution, and then the protein was digested with Trypsin Gold (Promega, Madison, WI, USA) with a ratio of protein: trypsin $=30: 1$ at $37^{\circ} \mathrm{C}$ for $16 \mathrm{~h}$. Peptides were reconstituted in 0.5 $\mathrm{M}$ TEAB and processed according to the manufacturer's protocol for 8-plex iTRAQ reagent (Applied Biosystems). Briefly, one unit of iTRAQ reagent was thawed and reconstituted in $24 \mu \mathrm{L}$ isopropanol. Samples were labeled with the iTRAQ tags as follows: control groups C1, C2, and C3 (tag 116, 117,115 , respectively) and experimental groups B1, B2, and B3 (tag 118, 119, and 121, respectively). The peptides were labeled with isobaric tags and incubated at room temperature for $2 \mathrm{~h}$. The iTRAQ-labeled peptide mixtures were reconstituted with $4 \mathrm{~mL}$ buffer A (25 mM NaH2PO4 in 25\% ACN, pH 2.7) and loaded onto a $4.6 \times 250 \mathrm{~mm}$ Ultremex SCX column containing $5-\mu \mathrm{m}$ particles (Phenomenex). The peptides were eluted at a flow rate of $1 \mathrm{~mL} / \mathrm{min}$ with a gradient of buffer A for $10 \mathrm{~min}, 5-60 \%$ buffer B (25 mM NaH2PO4, $1 \mathrm{M} \mathrm{KCl}$ in $25 \% \mathrm{ACN}, \mathrm{pH} 2.7$ ) for $27 \mathrm{~min}$, and $60-100 \%$ buffer B for $1 \mathrm{~min}$. The system was then maintained at $100 \%$ buffer B for 1 min before equilibrating with buffer A for $10 \mathrm{~min}$ prior to the next injection. Elution was monitored by measuring the absorbance at $214 \mathrm{~nm}$, and fractions were collected every $1 \mathrm{~min}$. The eluted peptides were pooled into 
20 fractions, desalted with a Strata X C18 column (Phenomenex) and vacuum-dried.

\subsubsection{LC-ESI-MS/MS analysis based on Q-EXACTIVE}

Each fraction was resuspended in buffer C (2\% ACN, 0.1\%FA) and centrifuged at $20000 \times \mathrm{g}$ for $10 \mathrm{~min}$; the final concentration of peptide was approximately $0.5 \mu \mathrm{g} / \mu \mathrm{L}$ on average. We loaded $10 \mu \mathrm{L}$ supernatant onto a LC-20AD nano HPLC (Shimadzu, Kyoto, Japan) by the auto sampler onto a $2 \mathrm{~cm}$ C18 trap column. The samples were loaded at $8 \mu \mathrm{L} / \mathrm{min}$ for $4 \mathrm{~min}$. Then, the gradient was run at $300 \mathrm{~nL} / \mathrm{min}$ starting from 2 to $35 \%$ of buffer D ( $98 \%$ ACN, $0.1 \%$ FA), followed by a 2 min linear gradient to $80 \%$ buffer D, maintenance at $80 \%$ buffer $\mathrm{D}$ for $4 \mathrm{~min}$, and finally a return to $5 \%$ in $1 \mathrm{~min}$.

The peptides were subjected to nano-electrospray ionization followed by tandem mass spectrometry (MS/MS) in a Q-EXACTIVE (Thermo Fisher Scientific, San Jose, CA) coupled online to the HPLC. Intact peptides were detected in the Orbitrap at a resolution of 70 000. A data-dependent procedure that alternated between one MS scan followed by 15 MS/MS scans was applied for the 15 most abundant precursor ions above a threshold ion count of 20000 in the MS survey scan with a following Dynamic Exclusion duration of $15 \mathrm{~s}$. The electrospray voltage applied was $1.6 \mathrm{kV}$. The AGC target for full MS was $3 \mathrm{e} 6$ and for MS2 was 1e5. For MS scans, the $m / z$ scan range was from 350 to 2000 Da. For MS2 scans, the $m / z$ scan range was $100-1800$.

\subsubsection{Data analysis}

Raw data files acquired from the Orbitrap were converted into MGF files using Proteome Discoverer 1.2 (PD 1.2, Thermo; 5600 msconverter), and the MGF files were then searched. Protein identification was performed by using the Mascot search engine (Matrix Science, London, UK; version 2.3.02) against the UniProt knowledge base containing 83425 protein sequences (http://www.uniprot.org/uniprot/?query = taxonomy:9606). For protein identification, a mass tolerance of $30 \mathrm{ppm}$ was permitted for intact peptide masses and 0.05 Da for fragmented ions, with allowance for one missed cleavage in the trypsin digests. Gln $\rightarrow$ pyro-Glu (N-term Q), oxidation $(\mathrm{M})$, and deamidation $(\mathrm{NQ})$ were tested as potential variable modifications, and carbamidomethyl (C), iTRAQ8plex (N-term), and iTRAQ 8plex (K) were tested as fixed modifications. The charge states of peptides were set to +2 and +3 . Specifically, an automatic decoy database search was performed in Mascot by choosing the decoy checkbox in which a random sequence of the database is generated and tested for raw spectra as well as the real database; each confident protein identification involves at least one unique peptide. For protein quantitation, it was required that a protein contain at least two unique spectra. The quantitative protein ratios were weighed and normalized by the median ratio in Mascot. We only used ratios with p-values $<0.05$; only fold changes $>1.2$ were considered significant.

\subsubsection{Bioinformatics analysis of proteomics data}

Identified proteins were classified according to annotations from the UniProt knowledge base (Swiss-Prot/TrEMBL, http://www.uniprot.org). Based on these proteins, an interaction network was constructed using the Web Search Tool for the Retrieval of Interacting Genes/Proteins (STRING, http://www.string-db.org), including both validated physical protein-protein interaction and putative interaction inferred from co-expression, gene fusion, and text mining, etc. Next, KEGG pathway enrichment analysis was performed with proteins in this network. The network was then imported into Cytoscape2.3.2 (www.cytoscape.org) [24] for topology analysis. The number of Directed Edges indicated the frequency of cross-talk in the network.

\subsection{Statistical analysis}

Results are expressed as the mean \pm standard deviation (SD). Student's $t$-test or one way ANOVA with repeated measures was used for statistical analyses (Graph Pad Prism, USA) of the data, where applicable. Unless otherwise specified, statistical analysis of the quantitative multiple group comparisons was assessed with ANOVA followed by Duncan's test (two sided). All tests were considered to be statistically significant when $P<0.05$.

\section{Results}

\subsection{Bupivacaine-induced cell injury in SH-SY5Y cells in a dose-dependent manner}

After the cells were exposed to bupivacaine $(0,0.5,1.0,1.5,2.0$, and $2.5 \mathrm{mM}$ ) for $24 \mathrm{~h}$, SH-SY5Y cell viability was determined with the Cell Counting Kit-8 and the lactate dehydrogenase (LDH) content was measured with an LDH Cytotoxicity Assay Kit. As shown in Fig. 1A, bupivacaine treatment (0, 0.5, $1.0,1.5,2.0$, and $2.5 \mathrm{mM}$ ) for $24 \mathrm{~h}$ decreased cell viability in a dose-dependent manner ranging from $0 \%$ to $92.73 \pm 12.18 \%$, and the IC50 of bupivacaine was $1.5 \mathrm{mM}$ (Fig. 1A). Bupivacaine exposure $(1.5,2.0$, and $2.5 \mathrm{mM})$ progressively and significantly increased cell injury, which manifested as elevated LDH release $(P<0.05$ versus $0 \mathrm{mM}$ bupivacaine; Fig. 1B). Therefore, we selected $1.5 \mathrm{mM}$ bupivacaine for use in our cell injury model in the ensuing studies $[1,2]$.

\subsection{Bupivacaine-induced apoptosis and DNA damage in SH-SY5Y cells}

Cell apoptosis in SH-SY5Y cells was determined by Hoechst 33258 and TUNEL staining. After incubation with $1.5 \mathrm{mM}$ 
A
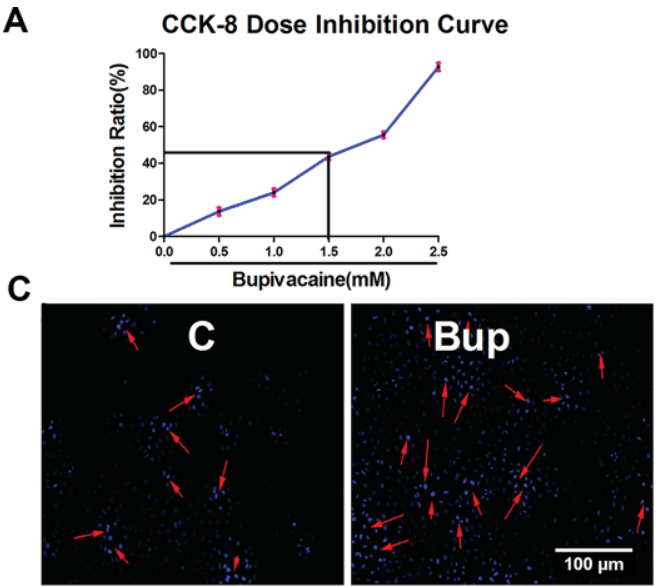

E

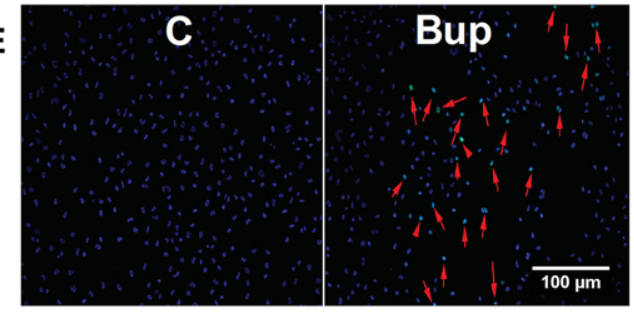

G
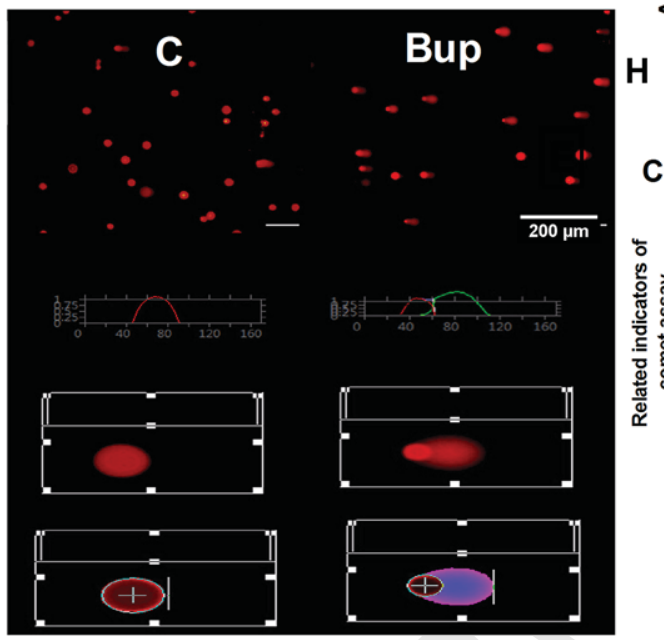

Comet Assay-DNA damage
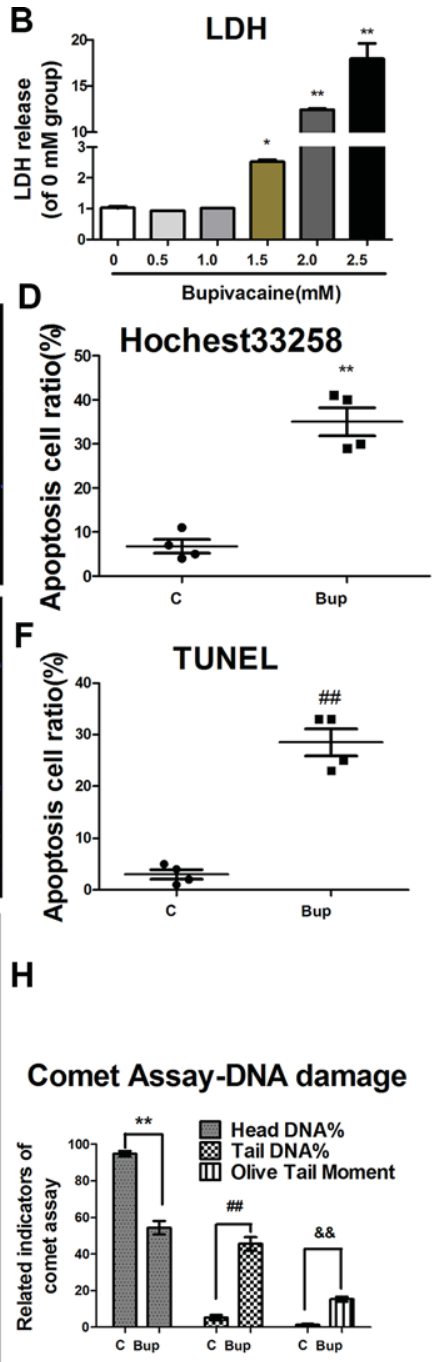

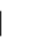

(2) deapivacall $24 \mathrm{~h}$ (IC50 death was assessed by lactate dehydrogenase (LDH) release after exposure to $(0,0.5,1.0$, $1.5,2.0$, and $2.5 \mathrm{mM}$ ) bupivacaine for $24 \mathrm{~h}$. (C, D) Nuclear condensation was determined by Hoechst 33258 staining after treating cells with $1.5 \mathrm{mM}$ bupivacaine for $24 \mathrm{~h}$. The arrows indicate condensed and coalesced nuclei with a brighter blue fluorescence (100 × magnification). (E, F) Cell apoptosis was detected by TUNEL staining after cells were exposed to $1.5 \mathrm{mM}$ bupivacaine for 24 h. $(G, H)$ An alkaline comet assay was used to test $1.5 \mathrm{mM}$ bupivacaine-induced cell DNA damage. $(\mathrm{H})$ The graph showed the analyzed results of comet assay-related indicators. Data are the mean \pm SD of three independent experiments performed in triplicate ${ }^{* *}, \#$, $\& \&=P<0.01$, versus the $\mathrm{C}$ group. bupivacaine for $24 \mathrm{~h}$, bupivacaine significantly increased cell apoptosis, as evidenced by the elevated number of condensed and coalesced nuclei and increased TUNEL positive cells in bupivacaine treated cells compared with the control group (Fig. 1C-F). As shown in Fig. 1G, DNA damage was determined by alkaline comet assay and showed that the nucleus of cells in the control group were nearly round. In bupivacainetreated cells, DNA fragment migration formed a comet with a small head and a large tail. These characteristics of DNA damage were further confirmed by the analyzed curves [the non-DNA damage cells showed a single peak (head DNA) while the DNA damage cells had double peaks (head DNA and tail DNA)]. In cells of the control group, a single peak was observed with a higher head DNA\% $(P<0.05$ versus bupivacaine group), while in the bupivacaine-treated cells, tail DNA \% and olive tail movement were increased and associated with decreased head DNA \% (Fig. $1 \mathrm{H} ; P<0.01$ ). These data indicated that $(1.5 \mathrm{mM})$ bupivacaine significantly caused DNA damage in SH-SY5Y cells.

\subsection{Bupivacaine induced oxidative stress and mitochondrial damage in SH-SY5Y cells}

To demonstrate the production of ROS after the cells were exposed to bupivacaine, we used DHE(dihydroethidium) as a probe to measure ROS. Bupivacaine treatment significantly increased cellular ROS production, as evidenced by the enhanced DHE red fluorescence (Fig. 2A and B). This was associated with MMP shown as a reduced red/green fluorescence ratio in JC-1 staining. This is a reflection of a reduction in 
A

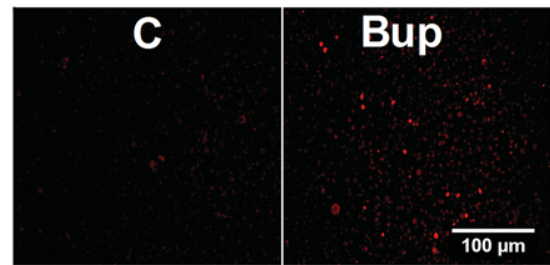

C

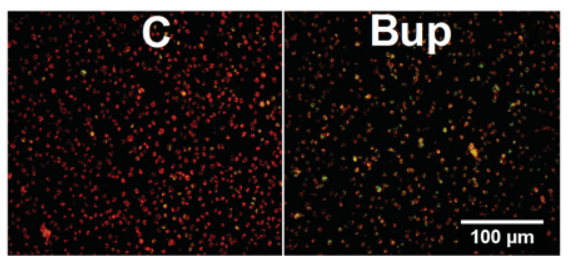

E

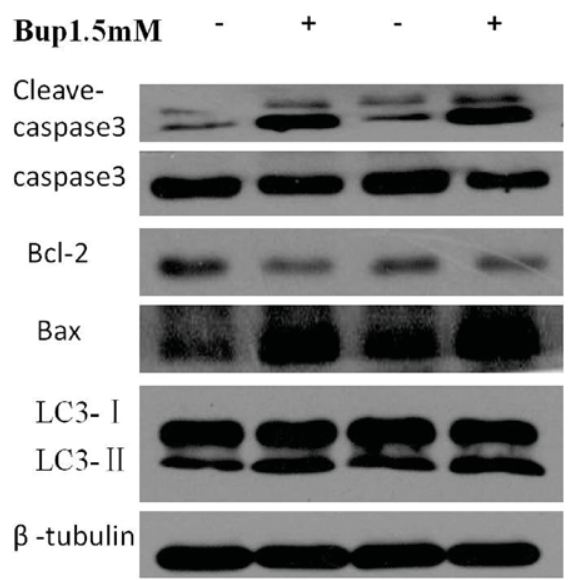

H

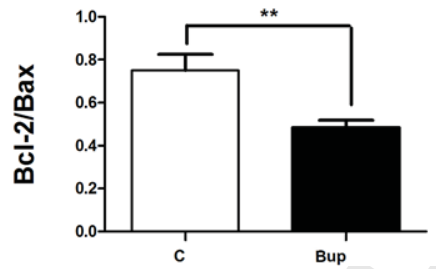

B

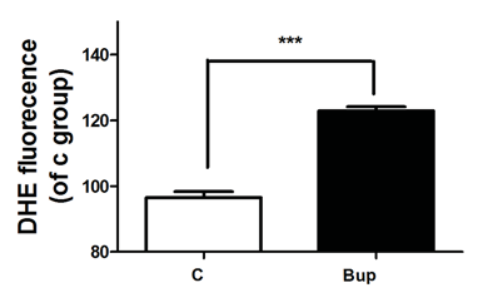

D

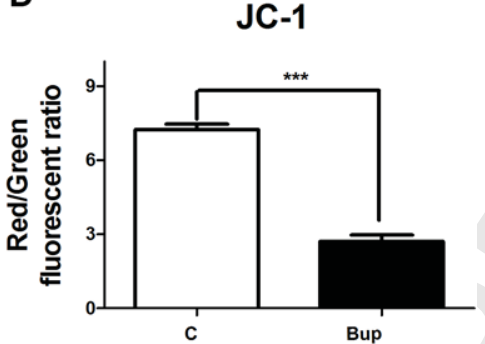

$\mathbf{F}$

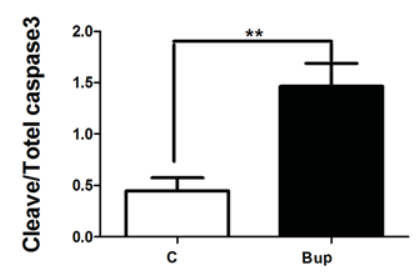

G

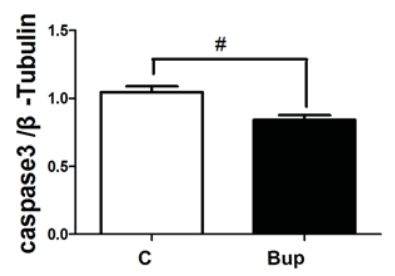

I

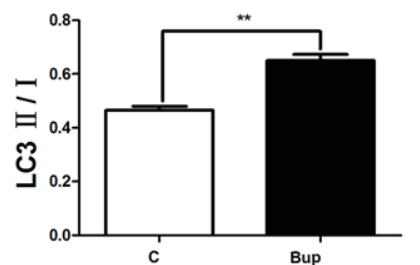

Figure 2. Bupivacaine induced oxidative stress and mitochondrial damage in $\mathrm{SH}-\mathrm{SY} 5 \mathrm{Y}$ cells. (A, B) Cellular ROS production was assessed by dihydroethidium (DHE) staining in $\mathrm{SH}$ SY5Y cells exposed to $1.5 \mathrm{mM}$ bupivacaine. Mitochondrial membrane permeability (MMP) loss was tested by JC-1 staining. (D) PanelC: control group cells showing that most cells had strong J-aggregation (red). Panel-B upper: $1.5 \mathrm{mM}$ bupivacaine treated cells showing that the majority of cells stained green due to low $\Delta \psi \mathrm{m}$. (C) The expression of apoptosis- and autophagy-related proteins was increased after SH-SY5Y cells were stimulated with $1.5 \mathrm{mM}$ bupivacaine for $24 \mathrm{~h}$. Bupivacaine downregulated the expression of total caspase-3 (G) and the $\mathrm{Bcl}-2 / \mathrm{Bax}$ ratio $(\mathrm{H})$, while increasing the level of cleaved/active caspase-3 (F) and the ratio of LC3II/I (I). Densitometry analysis was performed via normalization to $\beta$-tubulin. Data are the mean \pm SD of three independent experiments performed in triplicate $\left(^{* * *} P<0.001\right.$, $\# P<0.05,{ }^{* *} P<0.01$ versus $C$ group). mitochondrial transmembrane potential and mitochondrial damage (Fig. $2 \mathrm{C}$ and $\mathrm{D} ; \mathrm{P}<0.001$ versus $\mathrm{C}$ group).

\subsection{Bupivacaine activated apoptosis- and autophagy-related proteins in SH-SY5Y cells}

After stimulation with $(1.5 \mathrm{mM})$ bupivacaine for $24 \mathrm{~h}$, in bupivacaine-treated cells, the apoptosis-related protein expression of total caspase-3 (Fig. 2E and G) and the Bcl-2/Bax ratio (Fig. $2 \mathrm{E}$ and $\mathrm{H}$ ) were down-regulated, while the level of cleaved/active caspase 3 (Fig. $2 \mathrm{E}$ and F) were increased $(P$ $<0.05$ versus the $\mathrm{C}$ group). We next examine the changes in LC3 conversion, a marker for autophagosome forma- tion according to the guidelines for monitoring autophagy. The ratio of LC3 II/I (Fig. 2E and I) was significantly increased $(P<0.05$ versus the $\mathrm{C}$ group). These results indicated that bupivacaine may simultaneously activate apoptosis and autophagy.

\section{5 iTRAQ proteomics analysis}

A quantitative proteome analysis based on iTRAQ labeling was performed to explore changes in protein expression and intercommunications among pathways related to bupivacaine-induced neurotoxicity. To reduce the probability of false peptide identification, only peptides at a 95\% confidence interval by a MASCOT probability analysis greater than 
A

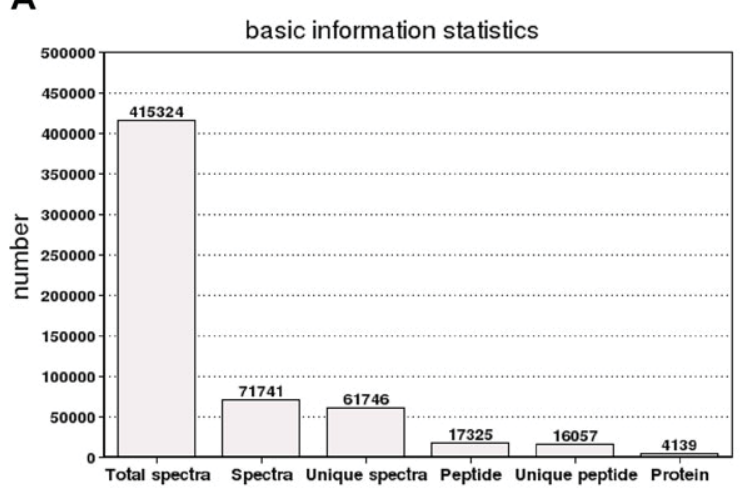

B
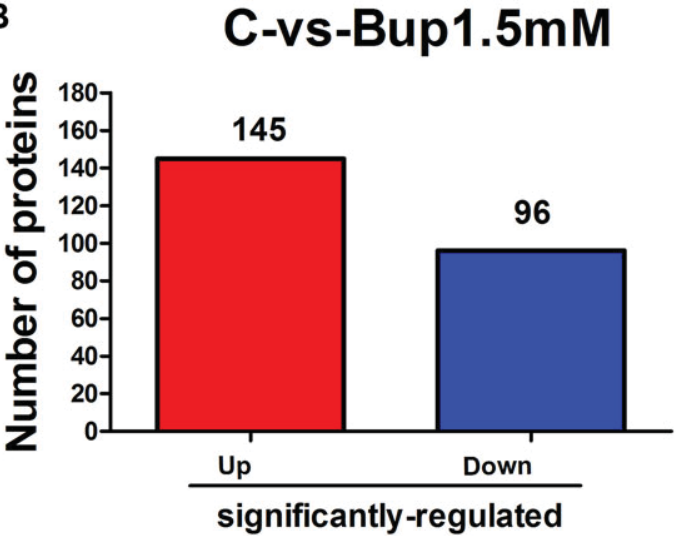

C

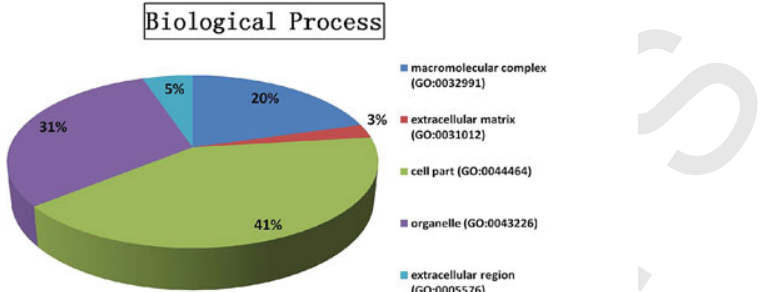

Figure 3. The total number of spectra, peptides, and identified proteins $(A)$ are shown. Of the total identified proteins, 241 proteins were significantly different between the $(1.5 \mathrm{mM})$ bupivacaine and $\mathrm{C}$ groups and included 145 upregulated and 96 downregulated proteins (B). Classification of the identified significant proteins. The biological processes (BPs), cellular components (CCs), and molecular functions (MFs) of the identified significant proteins were classified by the GO database (C).

"identity" were counted as identified. Each confident protein identification involved at least one unique peptide. For protein quantitation, it was required that a protein contain at least two unique spectra. The quantitative protein ratios were weighed and normalized by the median ratio in Mascot. We only used ratios with $p$-values $<0.05$, and only fold changes of $>1.2$ were considered as significant. A total of 415324 spectra (Fig. 3A) were obtained from the iTRAQ -LC-MS/MS proteomic analysis. After data filtering to eliminate lowscoring spectra, a total of 61746 unique spectra (Fig. 3A) were found to meet the strict confidence. A total of 4139 proteins (Fig. 3A) were identified and quantified at more than a $95 \%$ $\mathrm{CI}$, and proteins with no less than two unique peptides were considered to be a positive identification. Accordingly, 145 and 96 proteins were significantly up- and down-regulated, respectively, in the bupivacaine-treated group (Fig. 3B).

To expand on the molecular characterization of these identified significant proteins, the Uniport databases were searched to provide relevant information about their biological processes, cellular components, and molecular functions (Fig. 3C).
Furthermore, the cross-talk between these key networks involved many significant proteins and cell death pathways (apoptosis, mTOR, MAPK, PI3K, and insulin signaling). Interestingly, in accordance with cross-talk bioinformatics analysis, PI3K (PIK3CB and PIK3R2) was the most frequently targeted protein in each of the interactions (Fig. 4A and B).

\subsection{Validation of the expression of key proteins in the PI3K/Akt/FoxO1 and MAPK pathways by western blotting}

To confirm the results from our iTRAQ analysis, which showed a central role for PI3K in bupivacaine-induced neurotoxicity, we determined the expression of the key proteins in the PI3K/Akt/FoxO1 and MAPKs pathways. As shown in Fig. 5A and B, in SH-SY5Y cells, after bupivacaine stimulation, PI3K protein expression, Akt phosphorylation/activation (p-Akt), and FoxO1 phosphorylation/ activation (p-FoxO1) were significantly reduced $(P<0.01$ versus control). Moreover, the activation of Erk (p-Erk) was 


\section{A}

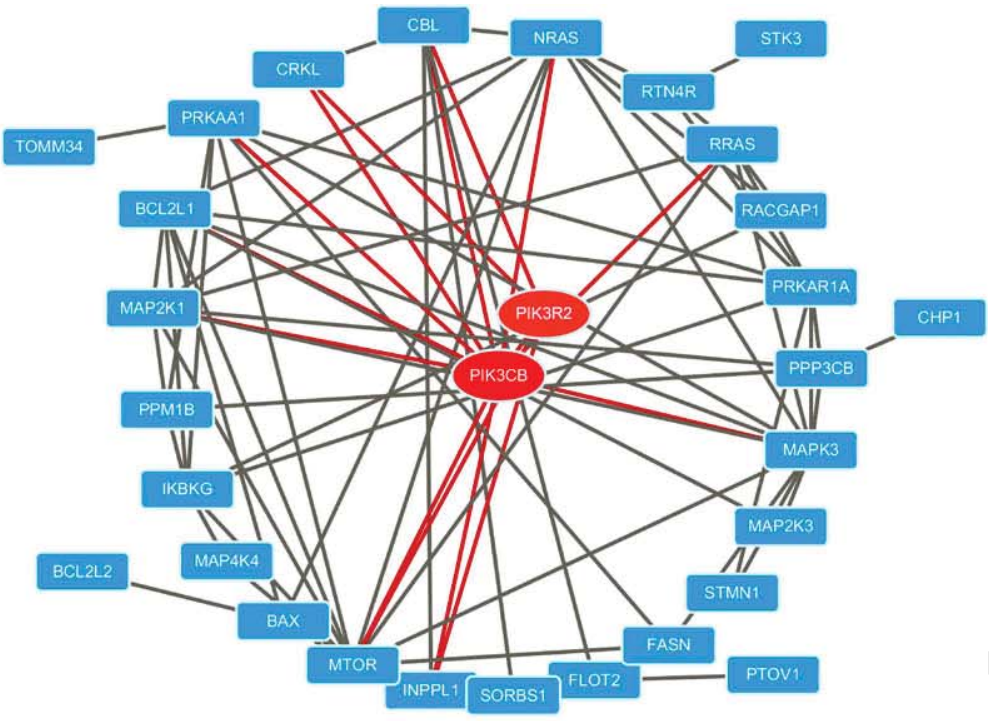

B

\begin{tabular}{|c|c|c|c|}
\hline Name & Number Of Directed Edges & NeighborhoodConnectivity & Radiality \\
\hline MTOR & 13 & 7 & 0.87931034 \\
\hline MAPK3 & 12 & 7.25 & 0.86781609 \\
\hline РІКЗСВ & 11 & 7. 90909091 & 0.87356322 \\
\hline NRAS & 10 & 8 & 0.87356322 \\
\hline PRKARIA & 9 & 7. 11111111 & 0.83908046 \\
\hline BCL2L1 & 9 & 8.44444444 & 0.84482759 \\
\hline PRKAA1 & 8 & 7.5 & 0.83908046 \\
\hline MAP2K1 & 8 & 8.875 & 0.83908046 \\
\hline RRAS & 7 & 8. 57142857 & 0.83333333 \\
\hline IKBKG & 7 & 6. 85714286 & 0.81034483 \\
\hline CBL & 7 & 5. 71428571 & 0.8045977 \\
\hline PPP3CB & 5 & 6.8 & 0.75862069 \\
\hline PIK3R2 & 5 & 7.8 & 0.7816092 \\
\hline INPPL1 & 5 & 7.8 & 0.7816092 \\
\hline RTN4R & 4 & 6.75 & 0.77586207 \\
\hline PPM1B & 4 & 7 & 0.74712644 \\
\hline BAX & 4 & 8.25 & 0.78735632 \\
\hline SORBS1 & 3 & 5 & 0.68390805 \\
\hline RACGAP1 & 3 & 8 & 0. 75862069 \\
\hline FLOT2 & 3 & 3. 66666667 & 0.66091954 \\
\hline FASN & 3 & 11 & 0.77011494 \\
\hline CRKL & 3 & 7. 66666667 & 0.74137931 \\
\hline STMN1 & 2 & 10.5 & 0.72988506 \\
\hline MAP4K4 & 2 & 10 & 0.74137931 \\
\hline MAP 2 K 3 & 2 & 10.5 & 0.72988506 \\
\hline Tомм34 & 1 & 8 & 0.67816092 \\
\hline STK3 & 1 & 4 & 0.61494253 \\
\hline PT0V1 & 1 & 3 & 0.5 \\
\hline CHP1 & 1 & 5 & 0.59770115 \\
\hline BCL2L2 & 1 & 4 & 0.62643678 \\
\hline
\end{tabular}

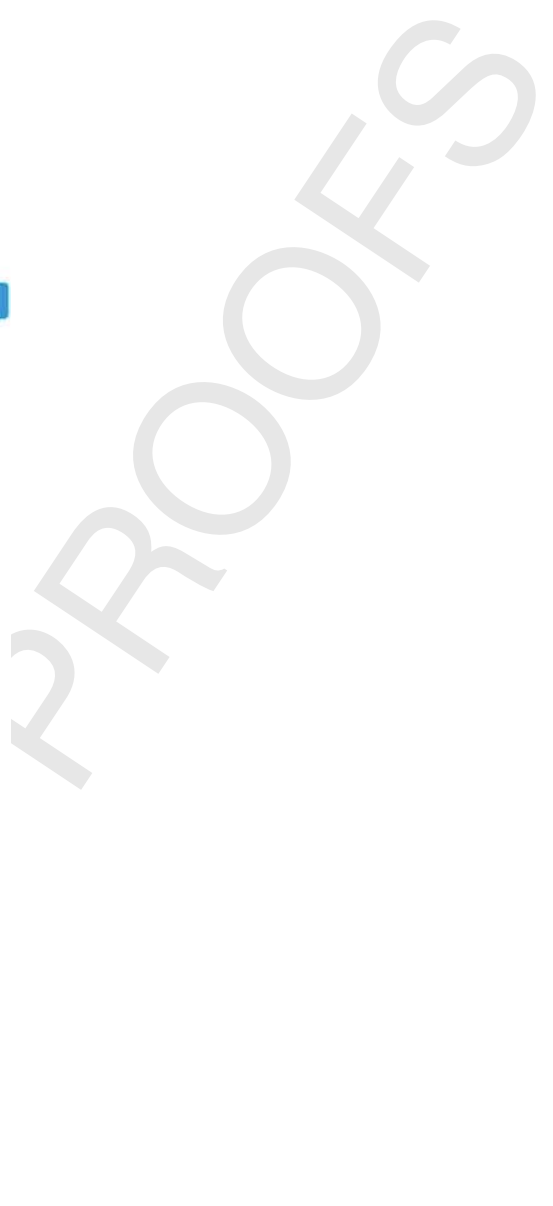

Figure 4. Subnetwork diagram of the PI3Kcentered protein interaction map. Proteinprotein interaction network analysis using STRING and the free software Cytoscape2.3.2 (www.cytoscape.org) revealed that the significant proteins from the bupivacaine-induced neurotoxicity-related signaling pathways (such as apoptosis, autophagy, MAPK, and mTOR) were highly associated with molecular PI3K. Additional interacting proteins are linked by grey and red lines (A). The number of Directed Edges indicated the frequency of cross-talk in the network (B). The isoforms of PI3K (PIK3CB and PIK3R2) had the most directed edges.

significantly reduced, while the activation of JNK (p-JNK) was significantly increased after bupivacaine exposure $(P<0.05$ versus the control; Fig. 5C-F).

\section{Discussion}

In this study, we showed that bupivacaine induced neurotoxicity by inducing cellular ROS overproduction, mitochondrial damage, DNA damage, neuronal apoptosis, and autophagy. Further, by using iTRAQ and bioinformatics analyses, we identified significant proteins in cell death related pathways for the first time in bupivacaine-treated cells (apoptosis, mTOR, MAPK, PI3K, and insulin) that were highly interactive and converged on the inactivation of PI3K, indicating that PI3K plays a central role in contacting and regulating different pathways related to bupivacaine-induced neurotoxicity.

Bupivacaine, an amide type local anesthetic, is one of the most widely used local anesthetics in clinics. Studies in animal and cellular models have proven that bupivacaine can be neurotoxic when applied to neural tissues at clinical concentrations [1,8] [9]. Multiple factors contribute to the 


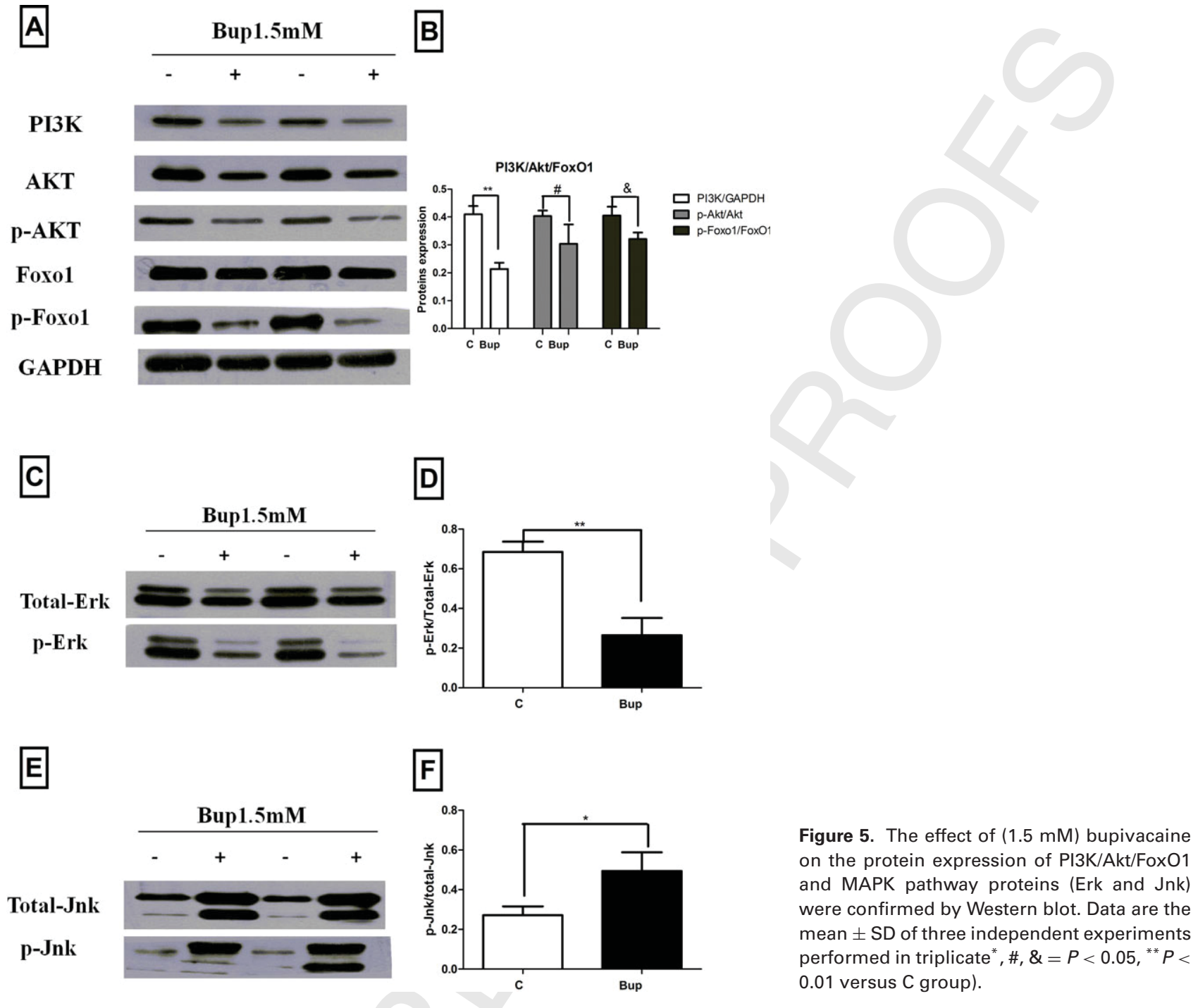

neurotoxicity of bupivacaine, including the overproduction of ROS and the impairment of autophagy, which all lead to neuronal cell apoptosis. In a Schwann cell model, bupivacaine induction increased ROS production and activated apoptosis-related proteins, resulting in cell apoptosis. These toxic effects of bupivacaine were attenuated by anti-oxidant treatment, indicating that ROS overproduction plays an important role in bupivacaine neurotoxicity. On the other hand, a recent study by $\mathrm{Li}$ et al. showed that bupivacaine induced toxicity by impairing autophagosome clearance in C2C12 cells, which was reversed by rapamycin (autophagy activator), implicating autophagy in bupivacaine-induced toxicity [9]. In our current study, the administration of bupivacaine in SH-SY5Y cells (human neuroblastoma cell line, were the established in vitro models that have been widely used for investigating the neurotoxicity of local anesthetics and other drugs-induced neurotoxicity, metabolism and signaling transduction pathways) induced the excess generation of ROS, which was associated with the activation of autophagy (manifested as an enhanced expression of LC3, an autophagosome marker) and apoptosis (enhanced expression of apoptosis related proteins: cleaved caspase 3 and the Bax/Bcl-2 ratio; Fig. 2). These results provide additional evidence for the involvement of ROS overproduction, the activation of autophagy, and the induction of apoptosis in the pathogenesis of bupivacaine-induced neurotoxicity.

Different signaling pathways have been proposed to underlie the neurotoxicity of bupivacaine, such as p38-MAPK, Erk, and JNK [12, 24-26]. Studies showed that cross-talk may exist among p-38 MAPK, Erk, and JNK [27] and that signaling pathways related to apoptosis may also be involved. However, to date, there are no studies that have investigated the interaction of these pathways in bupivacaine-induced neurotoxicity. In the current study, by employing iTRAQ proteomic and bioinformatics analyses, we showed, for the first time that the phosphoinositide 3 kinase (PI3K) pathway plays a 
central role in contacting and regulating different pathways in bupivacaine-induced neurotoxicity. Indeed, PI3K is considered one of the most important regulatory proteins, because it is involved in a series of different signaling pathways and regulates primary cellular functions including immunity, metabolism, and cardiac function $[28,29]$. Recent studies showed that the inhibition of PI3K signaling enhanced autophagy and thereby induced apoptosis [30-33]. Malcolm Campbell showed that there was significant cross-talk between the PI3K and MAPK pathways. Moreover, the inhibition of mTORC1-mediated activation of the MAPK pathway is PI3K-dependent [34]. PI3K also mediates the metabolic regulatory actions of insulin and insulin-induced gene expression for various cellular differentiation and proliferation events, which were mainly achieved through the activation of MAPK and Erk [35]. These results provide evidence that PI3K acts as a convergence point of signaling pathways in bupivacaineinduced neurotoxicity. In the current study, our iTRAQ proteomic analysis and bioinformatics analysis showed that, in bupivacaine-treated SH-SY5Y cells, PI3K was down-regulated and may be largely responsible for the alterations in signaling pathways including apoptosis, MAPK, mTOR, autophagy, and metabolic signaling (Fig. 5A and Supporting Information Table 1). Thus, an impairment in PI3K signaling may serve as the major mechanism that underlies the pathogenesis of bupivacaine-induced neurotoxicity. This concept was further confirmed in our study by determining the important downstream targets of the aforementioned signaling pathways, such as Akt, FoxO1, Erk, and JNK. Thus, our current study demonstrated that PI3K is central to signaling pathways that are highly involved in the pathomechanism of bupivacaineinduced neurotoxicity.

Interestingly, our iTRAQ proteomic analysis and bioinformatics analysis results further showed that there are two isoforms of PI3K, namely PIK3R2 and PIK3CB, that are involved in the PI3K-mediated neurotoxicity of bupivacaine (Fig. 5A). PI3Ks are a family of three classes of lipid kinases, which, on the basis of structural and biochemical characteristics, can be divided into class I enzymes, which are receptor-regulated PtdIns(4,5)P2 kinases; class II enzymes, which are PI3K-C2 kinases; and the class III enzyme, which is the PtdIns-specific enzyme Vps34. [29, 36]. However, the contribution of different PI3K isoforms to diverse organismal functions, as well as diseases or pathological conditions remains unclear. In the current study, in bupivacaine-treated SH-SY5Y cells, two isoforms (PIK3CB and PIK3R2) of PI3K were down-regulated. Interaction knowledge from STRING (both physical and nonphysical) suggested that the two PI3K isoforms (PIK3CB and PIK3R2) may have the potential to interact with distinct genes, indicating that different isoforms of PI3K may have different effects in the PI3K regulatory network. However, further studies and direct evidence are needed to investigate and distinguish the regulatory effects of different PI3K isoforms.

In conclusion, our current study demonstrated that bupivacaine exerts its neurotoxicity in SH-SY5Y cells by generating excess ROS and activating autophagy, which were associated with the modulation of signaling proteins that converge in PI3K (i.e., PIK3CB and PIK3R2), eventually leading to neuronal cell apoptosis. Thus, PI3K may serve as a novel target in the treatment of local anesthetic neurotoxicity and specifically bupivacaine-induced neurotoxicity. Effective strategies that can up-regulate PI3K, especially PIK3CB and PIK3R2, may attenuate bupivacaine-induced neurotoxicity in a clinical setting, and this concept merits further investigation in in vivo models of bupivacaine-induced neurotoxicity.

\section{Significance}

In the current study, we first showed that PI3K may play a central role in contacting and regulating the signaling pathways that contribute to bupivacaine-induced neurotoxicity. PI3K may serve as a novel target in the treatment of local anesthetic neurotoxicity in general and bupivacaine neurotoxicity in particular. Up-regulation of PI3K, especially PIK3CB and PIK3R2, may attenuate bupivacaine-induced neurotoxicity in a clinical setting.

This work was supported by grants from the National Natural Science Foundation of China (No. 81471272, No.81400995).

The authors have declared no conflicts of interest.

\section{References}

[1] Malet, A., Faure, M. O., Deletage, N., Pereira, B. et al., The comparative cytotoxic effects of different local anesthetics on a human neuroblastoma cell line. Anesth. Analg. 2015, 120, 589-596.

[2] Perez-Castro, R., Patel, S., Garavito-Aguilar, Z. V., Rosenberg, A. et al., Cytotoxicity of local anesthetics in human neuronal cells. Anesth. Analg. 2009, 108, 997-1007.

[3] Werdehausen, R., Fazeli, S., Braun, S., Hermanns, H. et al., Apoptosis induction by different local anaesthetics in a neuroblastoma cell line. Br. J. Anaesth. 2009, 103, 711-718.

[4] Lirk, P., Poroli, M., Rigaud, M., Fuchs, A. et al., Modulators of calcium influx regulate membrane excitability in rat dorsal root ganglion neurons. Anesth. Analg. 2008, 107, 673-685.

[5] Pollock, J. E., Neal, J. M., Stephenson, C. A., Wiley, C. E., Prospective study of the incidence of transient radicular irritation in patients undergoing spinal anesthesia. Anesthesiology 1996, 84, 1361-1367.

[6] Auroy, Y., Narchi, P., Messiah, A., Litt, L. et al., Serious complications related to regional anesthesia: results of a prospective survey in France. Anesthesiology 1997, 87, 479-486.

[7] Auroy, Y., Benhamou, D., Bargues, L., Ecoffey, C. et al., Major complications of regional anesthesia in France: the SOS Regional Anesthesia Hotline Service. Anesthesiology 2002, 97, 1274-1280.

[8] Li, D. F., Bahar, M., Cole, G., Rosen, M., Neurological toxicity of the subarachnoid infusion of bupivacaine, lignocaine or 2-chloroprocaine in the rat. Br. J. Anaesth. 1985, 57, 424-429. 
[9] Li, R., Ma, H., Zhang, X., Li, C. et al., Impaired autophagosome clearance contributes to local anesthetic bupivacaineinduced myotoxicity in mouse myoblasts. Anesthesiology 2015, 122, 595-605.

[10] Park, C. J., Park, S. A., Yoon, T. G., Lee, S. J. et al., Bupivacaine induces apoptosis via ROS in the Schwann cell line. J. Dent. Res. 2005, 84, 852-857.

[11] Johnson, M. E., Saenz, J. A., DaSilva, A. D., Uhl, C. B., Gores, G. J., Effect of local anesthetic on neuronal cytoplasmic calcium and plasma membrane lysis (necrosis) in a cell culture model. Anesthesiology 2002, 97, 1466-1476.

[12] Lirk, P., Haller, I., Colvin, H. P., Lang, L. et al., In vitro, inhibition of mitogen-activated protein kinase pathways protects against bupivacaine- and ropivacaine-induced neurotoxicity. Anesth. Analg. 2008, 106, 1456-1464, table of contents.

[13] Li, P., Shi, J., He, Q., Hu, Q. et al., Streptococcus pneumoniae induces autophagy through the inhibition of the $\mathrm{PI} 3 \mathrm{~K} / / \mathrm{Akt} / \mathrm{mTOR}$ pathway and ROS hypergeneration in $\mathrm{A} 549$ cells. Plos one 2015, 10, 00122753.

[14] Li, L., Zhang, Q.G., Lai, L.Y., Wen, X. J.etal., Neuroprotective effect of ginkgolide $B$ - on bupivacaine-induced apoptosis in SH-SY5Y cells. Oxidative Medicine Cellular Longevity 2013, 2013,159864 .

[15] Nikoletopoulou, V., Markaki, M., Palikaras, K., Tavernarakis, N., Crosstalk between apoptosis, necrosis and autophagy. Biochim. Biophys. Acta 2013, 1833, 3448-3459.

[16] Yusuf, M., Leung, K., Morris, K. J., Volpi, E. V., Comprehensive cytogenomic profile of the in vitro neuronal model SH-SY5Y. Neurogenetics 2013, 14, 63-70.

[17] Pahlman, S., Mamaeva, S., Meyerson, G., Mattsson, M E. et al., Human neuroblastoma cells in culture: a model for neuronal cell differentiation and function. Acta Physiol. Scand. Suppl. 1990, 592, 25-37.

[18] Craft, G. E., Chen, A., Nairn, A. C., Recent advances in quantitative neuroproteomics. Methods 2013, 61, 186-218.

[19] An, K., Fang, L., Luo, R., Wang, D. et al., Quantitative Proteomic analysis reveals that transmissible gastroenteritis virus activates the JAK-STAT1 signaling pathway. J. Proteome Res. 2014, 13, 5376-5390.

[20] Chen, H., Xu, L., Yin, L., Xu, Y. et al., iTRAQ-based proteomic analysis of dioscin on human HCT-116 colon cancer cells. Proteomics 2014, 14, 51-73.

[21] Xu, J., Li, H., Irwin, M. G., Xia, Z. Y. et al., Propofol ameliorates hyperglycemia-induced cardiac hypertrophy and dysfunction via heme oxygenase-1/signal transducer and activator of transcription 3 signaling pathway in rats. Crit. Care Med. 2014, 42, e583-594.

[22] Li, H., Yao, W., Irwin, M. G., Wang, T. et al., Adiponectin ameliorates hyperglycemia-induced cardiac hypertrophy and dysfunction by concomitantly activating Nrf2 and Brg1. Free Radic.Biol. Med. 2015, 84, 311-321.
[23] Mao, X., Wang, T., Liu, Y., lrwin, M. G. et al., N acetyleysteine and allopurinol confer synergy in attenuating myocardial isehemia injury via restoring HIF-1alpha/HO-1 signaling in diabetic rats. Plos one 2013, 8, e68949.

[24] Lu, J., Xu, S. Y., Zhang, Q. G., Xu, R., Lei, H. Y., Bupivacaine induces apoptosis via mitochondria and p38 MAPK dependent pathways. Eur. J. Pharmacol. 2011, 657, 51-58.

[25] Lu, J., Xu, S. Y., Zhang, O. G., Lei, H. Y., Bupivacaine induces reactive oxygen species production via activation of the AMP-activated protein kinase-dependent pathway. Pharmacology 2011, 87, 121-129.

[26] Wen, $X_{n}, X_{u}, S_{\text {., Liu, }} H_{\text {, }}$ Zhang, $Q$. et al., Neurotoxicity induced by bupivacaine via $T$ type calcium channels in SH-SY5Y cells. PloS-one 2013, 8, e62942.

[27] Mauro, A., Ciccarelli, C., De Cesaris, P., Scoglio, A. et al., PKCalpha-mediated ERK, JNK and p38 activation regulates the myogenic program in human rhabdomyosarcoma cells. J. Cell Sci. 2002, 115, 3587-3599.

[28] Krasilnikov, M. A., Phosphatidylinositol-3 kinase dependent pathways: the role in control of cell growth, survival, and malignant transformation. Biochemistry (Mosc.) 2000, 65, 59-67.

[29] Vanhaesebroeck, B., Stephens, L., Hawkins, P., PI3K signalling: the path to discovery and understanding. Nat. Rev. Mol. Cell Biol. 2012, 13, 195-203.

[30] Saiki, S., Sasazawa, Y., Imamichi, Y., Kawajiri, S. et al., Caffeine induces apoptosis by enhancement of autophagy via $\mathrm{PI3K} / \mathrm{Akt} / \mathrm{mTOR} / \mathrm{p} 70$ S6K inhibition. Autophagy 2014, 7, 176187.

[31] Kumar, D., Shankar, S., Srivastava, R. K., Rottlerin induces autophagy and apoptosis in prostate cancer stem cells via $\mathrm{PI} 3 \mathrm{~K} / \mathrm{Akt} / \mathrm{mTOR}$ signaling pathway. Cancer Lett. 2014, 343, 179-189.

[32] Roy, R., Singh, S. K., Chauhan, L. K., Das, M. et al., Zinc oxide nanoparticles induce apoptosis by enhancement of autophagy via PI3K/Akt/mTOR inhibition. Toxicol. Lett. 2014, 227, 29-40.

[33] Sekulic, A., Hudson, C. C., Homme, J. L., Yin, P. et al., A direct linkage between the phosphoinositide 3-kinase-AKT signaling pathway and the mammalian target of rapamycin in mitogen-stimulated and transformed cells. Cancer Res. $2000,60,3504-3513$.

[34] Carracedo, A., Ma, L., Teruya-Feldstein, J., Rojo, F. et al., Inhibition of mTORC1 leads to MAPK pathway activation through a PI3K-dependent feedback loop in human cancer. J. Clin. Invest. 2008, 118, 3065-3074.

[35] Rains, J. L., Jain, S. K., Oxidative stress, insulin signaling, and diabetes. Free Radic. Biol. Med. 2011, 50, 567-575.

[36] Kong, D., Zhang, Y., Yamori, T., Duan, H., Jin, M., Inhibitory activity of flavonoids against class I phosphatidylinositol 3-kinase isoforms. Molecules 2011, 16, 5159-5167. 